\title{
Cross-reaction of POC-CCA urine test for detection of Schistosoma mekongi in Lao PDR: a cross-sectional study
}

\author{
Anousin Homsana ${ }^{1,2,3}$, Peter Odermatt ${ }^{2,3}$, Phonesavanh Southisavath ${ }^{1,2,3,4}$, Aya Yajima ${ }^{5}$ and Somphou Sayasone ${ }^{1 *}$ (D)
}

\begin{abstract}
Background: The point-of-care circulating cathodic antigen (POC-CCA) test is increasingly used as a rapid diagnostic method for Schistosoma mansoni infection. The test has good sensitivity, although false positive results have been reported among pregnant women and patients with urine infections and hematuria. We validated the POC-CCA test's ability to diagnose Schistosoma mekongi infection in Lao People's Democratic Republic (Lao PDR), where S. mekongi is endemic. Of particular interest was the test's specificity and possible cross-reactivity with other helminth infections.
\end{abstract}

Methods: We conducted a cross-sectional study of children and adults in the provinces of Champasack (Schistosoma mekongi and Opisthorchis viverrini endemic), Savannakhet (O. viverrini endemic) and Luang Prabang (soil-transmitted helminths endemic) between October 2018 and April 2019. POC-CCA and urine dipstick tests were administered to all study participants, while an additional pregnancy test was offered to women. Two stool samples were collected from participants and examined with a Kato-Katz test (two smears per stool). Logistic regression was used to associate potential confounding factors (predictors) with POC-CCA test results (outcome).

Results: In S. mekongi-endemic Champasack, 11.5\% $(n=366)$ and $0.5 \%(n=2)$ of study participants had positive POC-CCA and Kato-Katz test results, respectively. Only one of the two Kato-Katz positive patients was also POC-CCA positive. In Champasack and Luang Prabang, where S. mekongi is not endemic, the POC-CCA test yielded (presumably) false positive results for $6.0 \%(n=22)$ and $2.5 \%(n=9)$ of study participants, respectively, while all of the Kato-Katz tests were negative. POC-CCA positive test results were significantly associated with $O$. viverrini infection (1.69, 95\% confidence interval $(C): 1.02-2.77, P=0.042)$, increased leukocytes (adjusted Odds Ratio $(a O R)=$ $1.58,95 \% \mathrm{Cl}: 1.15-2.17, P=0.005)$ and hematuria $(\mathrm{aO} R=1.50,95 \% \mathrm{Cl}: 1.07-2.10, P=0.019)$ if the observed trace was counted as a positive test result. Two pregnant women from Champasack province had POC-CCA positive tests.

Conclusions: We observed a cross-reaction between the POC-CCA test and O. viverrini infection. To some extent, we can confirm previous observations asserting that POC-CCA provides false positive results among patients with urinary tract infections and hematuria. In S. mekongi-endemic areas, POC-CCA can be applied cautiously for surveillance purposes, keeping in mind the considerable risk of false positive results and its unknown sensitivity.

Keywords: Point-of-care circulating cathodic antigen, Lao People's Democratic Republic, Kato-Katz, Schistosoma mekongi, Opisthorchis viverrini, Soil-transmitted helminth

\footnotetext{
* Correspondence: somphou.sayasone@yahoo.com

'Lao Tropical and Public Health Institute, Ministry of Health, Vientiane Capital, Lao People's Democratic Republic

Full list of author information is available at the end of the article
}

\section{$\triangle B M C$}

(c) The Author(s). 2020 Open Access This article is licensed under a Creative Commons Attribution 4.0 International License, which permits use, sharing, adaptation, distribution and reproduction in any medium or format, as long as you give appropriate credit to the original author(s) and the source, provide a link to the Creative Commons licence, and indicate if changes were made. The images or other third party material in this article are included in the article's Creative Commons licence, unless indicated otherwise in a credit line to the material. If material is not included in the article's Creative Commons licence and your intended use is not permitted by statutory regulation or exceeds the permitted use, you will need to obtain permission directly from the copyright holder. To view a copy of this licence, visit http://creativecommons.org/licenses/by/4.0/ The Creative Commons Public Domain Dedication waiver (http://creativecommons.org/publicdomain/zero/1.0/) applies to the data made available in this article, unless otherwise stated in a credit line to the data. 


\section{Introduction}

The schistosomiasis, caused by the Schistosoma mekongi blood fluke, affects communities in the Mekong River Basin, particularly those in southern Lao People's Democratic Republic (Lao PDR) and in northern Cambodia [1]. The parasite was first discovered in 1957, in a Lao patient hospitalized in Paris, France [2]. In the 1980s, the public health problem posed by the infection was recognized in two districts (Khong and Mounlapamok) in Champasack province, Lao PDR. The first round of community-based chemotherapy intervention was implemented from 1989 to 1999; a second round started in 2007, and continues to this day $[3,4]$. As a result, the prevalence of S. mekongi infection and heavy infection intensity in the two districts has fallen to less than $5 \%$ and $1 \%$, respectively [5].

In 2016, the Ministry of Health (MOH) in Lao PDR adopted a national action plan for eliminating schistosomiasis in the two endemic districts, with the goals of eliminating schistosomiasis as a public health problem by 2018 , interrupting transmission by 2025 and verifying elimination by 2030 [5]. To this end, a surveillance system was set up to monitor the progress of intervention. It includes annually monitoring $S$. mekongi infection via village sentinel sites and rotating village spot checks. Currently, the gold standard for detecting intestinal schistosomiasis, recommended by the World Health Organization (WHO), is the Kato Katz (KK) test, which detects eggs in stool by staining a sieved fecal sample and examining it under a microscope [5]. As such, the Lao PDR national schistosomiasis elimination program continues to rely on this method to monitor S. mekongi infection. However, it is widely recognized that the Kato-Katz technique is not particularly sensitive to light-intensity infections; hence, more sensitive diagnostic tools are urgently required to continue monitoring the impacts of interventions in low-prevalence settings and, ultimately, to verify the absence of schistosomiasis transmission [6].

Recently, more sensitive diagnostic tools have been developed to detect circulating antigens of schistosomes in the host's blood and urine $[7,8]$. One such tool is the rapid point-of-care test to detect circulating cathodic $S$. mansoni antigens (POC-CCA) in urine [7]. In a study of asymptomatic Eritrean refugees from $S$. mansoni-endemic areas, POC-CCA test in urine showed high sensitivity (91.3\%) [9]. In 2015, WHO recommended the use of POC-CCA for mapping S. mansoni endemicity in affected countries [10].

The S. mansoni POC-CCA test was applied in $S$. mekongi-endemic areas of Lao PDR (Khong district, Champasack province) and Cambodia (Kratié province) in the year 2017 [11]. The study found that while the KK test detected S. mekongi eggs in $6.4 \%$ of the 377 study participants using three samples per participant, $21.0 \%$ of the study participants tested positive with POC-CCA. If trace observations were also considered positive, $53.9 \%$ of study participants would have been considered infected [11]. However, when urine POC-CCA and KK results were compared to the overall composite assessment reference, POC-CCA sensitivity was only $24.1 \%$ but still almost two times higher than that of the KK method (13.6\%) [11].

A further study to validate the POC-CCA urine test in asymptomatic Eritrean refugees from $S$. mansoni-affected areas confirmed the high sensitivity of $90 \%$, but showed a relatively low specificity of $73.6 \%$ [12]. A follow-up study of individuals who had never visited a Schistosoma-endemic area (residents of Switzerland), indicated a considerable amount of false positive (low specificity) cases among healthy adults (80\% specificity), infants under three years ( $83 \%$ specificity), pregnant women ( $80 \%$ specificity), patients with urinary tract infections (73\% specificity), patients with hematuria (93\% specificity) and patients with bladder malignancies ( $80 \%$ specificity) [13]. This raised a concern about the use of POC-CCA in Southeast Asian settings where many other parasitic diseases are coendemic, especially those caused by trematode species, which might cross-react with POC-CCA.

The concerns regarding the test's low specificity and potential cross-reactions with other highly prevalent and related trematode species, particularly Opisthorchis viverrini and different minute intestinal fluke (MIF) species in Lao PDR, warranted further validation of the test. We assessed the performance of the $S$. mansoni POC-CCA urine test in S. mekongi-endemic areas and non-endemic areas of Lao PDR, in terms of specificity and potential cross-reaction with other helminth infections and pathologies.

\section{Materials and methods \\ Study area and population}

This study was conducted in three different helminthendemic settings in Lao PDR, specifically one district per province in Champasack, Savannakhet and Luang Prabang. Khong district, in Champasack province, is located in the southern end of the country (geographical coordinates: 14.094281, 105.792992). Figure 1 shows a map of the study location. It borders Cambodia and has a population of 80000 [14]. S. mekongi, O. viverrini and soiltransmitted helminth $(\mathrm{STH})$ are known to be endemic in this district [15]. Champhone district, in Savannakhet province, is situated in the central part of the country (geographical coordinates: 16.528993, 105.234703) and is populated by some 100000 people [14]. In this area for $O$. viverrini, intestinal trematodes and STH [16] but not for S. mekongi. Namback District, in Luang Prabang Province, is in the northern part of Lao PDR (geographical coordinates: 20.630490, 102.467147) and has a total of 68863 people [14]. Namback is highly endemic for $\mathrm{STH}$, but not for S. mekongi nor O. viverrini [17]. 


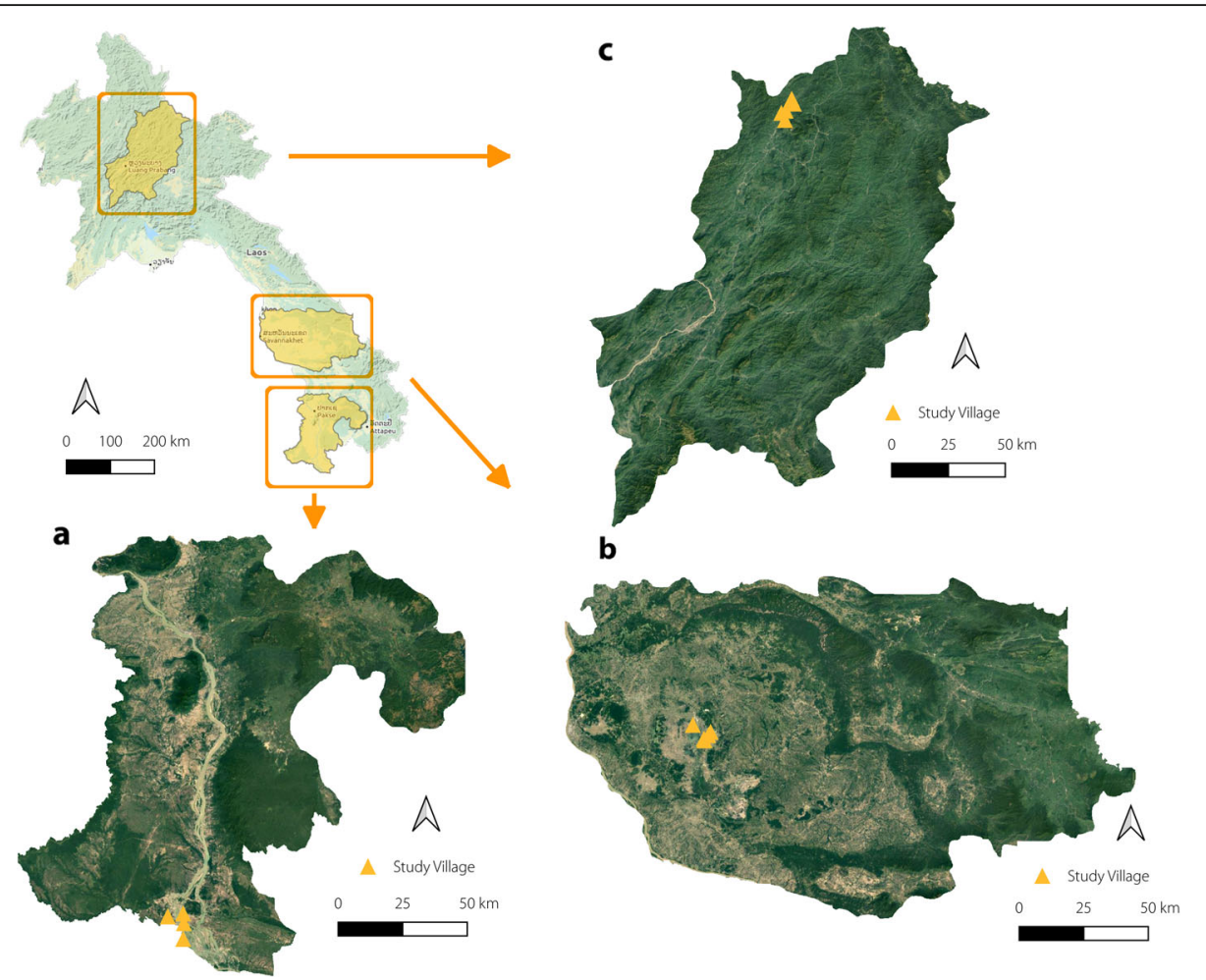

Fig. 1 Map of Lao PDR and study provinces and villages in a) Champasack, b) Savannakhet and c) Luang Prabang provinces. (sources: OpenStreetMap contributors, 2015; Map data@2015 google)

\section{Study design}

For this cross-sectional study, we used purposive sampling. Five study villages and five primary schools were selected in each district. In each village selected, about 40-60 adults aged 35 years and older were invited to participate. The selection process went as follows: (i) village demographic information was obtained from the village register, available at the district health offices; (ii) study households in each village were contacted with the help of the village leaders, while the heads of primary schools provided student lists; and (iii) all adult members of the selected household, aged 35 years and older and at home on the days of study were invited to participate. Primary school students were also invited to participate. From each primary school, 30-40 students from grades 3 to 5 were included in the study.

The study was carried out between October 2018 and April 2019 (from October to December 2018 in Champasack; between January and February 2019 in Savannakhet; and between March and April 2019 in Luang Prabang).

\section{Field and laboratory procedures Stool examination}

Each study participant was asked to provide two stool samples on two consecutive days for parasitological analysis using the KK method. Two KK smears were prepared from each stool sample (total four KK smears per person). The smears were allowed to clear for 30 to 60 min prior to examination under a light microscope, conducted by experienced microscopists. All helminth eggs were counted and recorded for each species separately.

\section{POC-CCA strip}

A 20-centiliter urine sample was collected from each study participant and examined immediately. The POCCCA strip (Schistosome POC-CCA Test, ICT Diagnostics, Cape Town South Africa) required $100 \mu \mathrm{l}$ urine and the test was read after $20 \mathrm{~min}$. The results were interpreted and recorded according to the manufacturer's instructions on test band intensity, i.e. positive when the test yields a pink band, trace positive when the test band is barely visible, and negative when no band appears [18].

\section{Urine analysis}

Additional urine analysis was performed using a dipstick (Combur 10, Roche, Germany). Blood in the urine (hematuria), leukocyte and nitrite, as well as protein (proteinuria) are among the 10 signs suggestive of a urinary tract infection $[19,20]$. The test strip was dipped in the urine sample and the result was read after $1 \mathrm{~min}$, according to the manufacturer's instructions. The intensity of the reaction zone was compared to the color label 


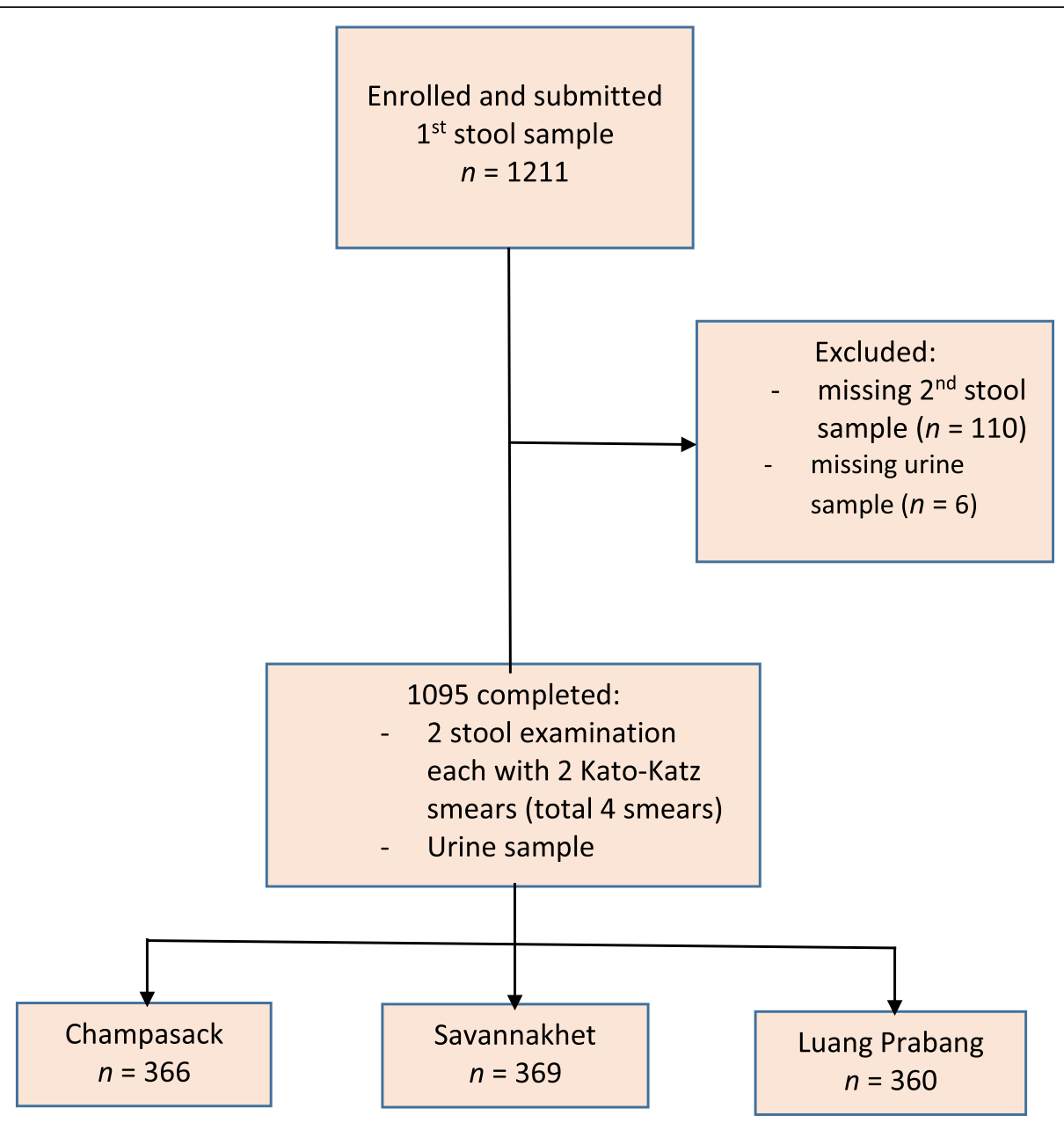

Fig. 2 Study enrollment

provided on the dipstick box. Categorical numbers correspond to the substance concentration in the urine sample, thereby providing cut-off values for positive or negative results. For example, leucocytes is classified as negative $(<$ $10 \mathrm{leu} / \mu \mathrm{l}), 1+(\sim 10-25 \mathrm{leu} / \mu \mathrm{l}), 2+(\sim 75-500 \mathrm{leu} / \mu \mathrm{l})$, or $3+$ $(\sim 500 \mathrm{leu} / \mu \mathrm{l})$; nitrite is negative (detection limit $<0.5 \mathrm{mg}$ / L) or positive; blood erythrocyte is negative, $1+(\geq 10$ $25 \mathrm{ery} / \mu \mathrm{l}), 2+(\geq 25-50 \mathrm{ery} / \mu \mathrm{l}), 3+(\geq 50-250 \mathrm{ery} / \mu \mathrm{l})$ or $4+$ $(\geq 250 \mathrm{ery} / \mu \mathrm{l}))$; while proteinuria can be negative $(<0.1 \mathrm{~g} /$ $\mathrm{L}), 1+(0.3 \mathrm{~g} / \mathrm{L}), 2+(1 \mathrm{~g} / \mathrm{L})$, or $3+(5 \mathrm{~g} / \mathrm{L}))[21]$.

A pregnancy test (One Step Pregnancy Test, AI Diagnostic, Shandong, China) was performed with samples from all female participants between 12 and 45 years of age.

\section{Data management and statistical analysis}

Data were collected and stored using electronic tablets. The questionnaires and forms had been developed in the Commcare server (www.commcarehq.org). Commcare ODK, version 3.4, was installed on the tablets for field data collection. The completed forms were converted to Microsoft Excel version 16 (Microsoft Cooperation,
Washington, USA) files and checked for completeness and consistency.

Only individuals with complete datasets were included in the final analysis. All statistical analysis was performed using STATA (version 16, Stata Cooperation, Texas, USA).

Descriptive statistics (frequency, proportions and means) were applied to describe the study participants' socio-demographic characteristics, infection status and infection intensity. The results of the POCCCA urine test for the detection of S. mekongi was classified into one of two scenarios: (i) a reliable scenario, where a trace observation was considered as a negative result; and (ii) a doubtful scenario, where a trace observation was considered as a positive result. A bivariate logistic regression model was used to associate the results of the POC-CCA urine test (outcome) with covariates, such as study locations, demographic information, helminth infections, and urine analysis results (the presence of leukocytes and/ or nitrites were combined into one variable indicating urinary tract infection). The crude odds ratio (cOR) 
and 95\% confidence intervals $(95 \% C I)$ were calculated. Variables with a significance level less than $20 \%$ were included in the multivariate logistic regression analysis. The adjusted odds ratio (aOR) and 95\% CI were calculated. All variables with $P$-values less than 0.05 were considered statistically significant.

\section{Results}

\section{Enrollment of study participants}

Of the household members and school children who had been informed of our study, 1211 individuals were enrolled and submitted stool samples. In total, 1095 participants completed all requirements and were included in the final analysis; 366, 369, and 360 participants came from Champasack, Savannakhet and Luang Prabang, respectively (Fig. 2).

\section{Socio-demographic characteristics of the study participants}

The socio-demographic characteristics of the study participants from all three study sites were collected, as shown in Table 1. Slightly more than half of the participants were females (54.4\%). The study population consisted of children below 13 years of age $(33.8 \%)$ and adults aged 35 years or older (66.2\%). The proportion of adult participants was slightly higher in Champasack (56.8\%) and in Savannakhet (56.4\%). However, in Luang Prabang, only $14.5 \%$ were children. The majority of participants (67.1\%) belonged to the Lao-Tai ethnic group. Most participants had finished primary school (70.9\%) and were farmers $(62.2 \%)$.

\section{Helminth infection diagnosed by Kato-Katz test}

The results of the KK stool examination were summarized in Table 2 . Only two study participants $(0.5 \%)$ were found to be infected with $S$. mekongi. Both were from $S$. mekongi endemic villages in Champasack. In the two other provinces (Savannakhet and Luang Prabang), no $S$. mekongi infection was detected. The prevalence of $O$. viverrini infection was very high in Champasack (62.9\%) and in Savannakhet (77.2\%), and low in Luang Prabang (12.2\%). The prevalence of hookworm infection was high in all three study sites: $40.4 \%$ in Champasack; $68.6 \%$ in Savannakhet, and $86.4 \%$ in Luang Prabang. STH infections, namely $T$. trichiura and $A$. lumbricoides, were rarely diagnosed in Champasack $(0.5$ and $0.2 \%$, respectively) and Savannakhet (1.4 and $0.3 \%)$, but were highly prevalent in Luang Prabang (31.7 and 12.5\%).

Table 1 Socio-demographic characteristics of the study participants

\begin{tabular}{|c|c|c|c|c|}
\hline Indicators & $\begin{array}{l}\text { Champasack, } n=366 \\
\%(\boldsymbol{n})\end{array}$ & $\begin{array}{l}\text { Savannakhet, } n=369 \\
, \%(\boldsymbol{n})\end{array}$ & $\begin{array}{l}\text { Luang Prabang, } n=360 \\
, \%(\boldsymbol{n})\end{array}$ & $\begin{array}{l}\text { Total, } n=1059 \\
, \%(\boldsymbol{n})\end{array}$ \\
\hline \multicolumn{5}{|l|}{ Gender } \\
\hline Male & $45.9(168)$ & $42.5(157)$ & $48.3(174)$ & 45.6 (499) \\
\hline Female & $54.1(198)$ & $57.5(212)$ & $51.7(186)$ & $54.4(596)$ \\
\hline \multicolumn{5}{|l|}{ Age in year } \\
\hline $\begin{array}{l}\leq 13 \\
\text { Mean }(\min -\max )\end{array}$ & $\begin{array}{l}43.2(158) \\
9.5(8-13)\end{array}$ & $\begin{array}{l}43.6(161) \\
9.5(7-13)\end{array}$ & $\begin{array}{l}14.2(51) \\
10.3(8-13)\end{array}$ & $\begin{array}{l}33.8(307) \\
9.4(7-13)\end{array}$ \\
\hline$\geq 35$ & $56.8(208)$ & $56.4(208)$ & 85.8 (309) & $66.2(725)$ \\
\hline Mean (min - max) & $50.1(35-77)$ & $52.7(35-83)$ & $47.2(35-60)$ & $49.6(35-83)$ \\
\hline \multicolumn{5}{|l|}{ Ethnicity } \\
\hline Lao-Tai & $100(366)$ & $100(369)$ & $0(0)$ & $67.1(735)$ \\
\hline Minority (Lao-Theung) & $0(0)$ & $0(0)$ & $100(360)$ & $32.9(360)$ \\
\hline \multicolumn{5}{|l|}{ Education } \\
\hline Illiterate & $5.7(21)$ & $10.7(38)$ & $31.9(115)$ & 15.9 (174) \\
\hline Primary & 78.7 (288) & $70.7(261)$ & $63.3(228)$ & 70.9 (777) \\
\hline Secondary & $15.6(57)$ & $16.3(60)$ & $4.7(17)$ & $12.2(134)$ \\
\hline University & $0(0)$ & $2.7(10)$ & $0(0)$ & $0.9(10)$ \\
\hline \multicolumn{5}{|l|}{ Profession } \\
\hline Farmer & 53.5 (196) & $53.7(198)$ & 85.8 (309) & $64.2(703)$ \\
\hline Student & $43.2(158)$ & $43.6(161)$ & $14.2(51)$ & $33.8(307)$ \\
\hline Fisherman & $3.3(12)$ & $0(0)$ & $0(0)$ & $1.1(12)$ \\
\hline Teacher & $0(0)$ & $2.7(10)$ & $0(0)$ & $0.9(10)$ \\
\hline
\end{tabular}


Table 2 Results of Kato-Katz stool examination: Prevalence and intensity of helminth infections

\begin{tabular}{|c|c|c|c|c|c|c|c|c|}
\hline \multirow[t]{2}{*}{ Parasites $^{\mathbf{a}}$} & \multicolumn{2}{|c|}{ Champasack, $\boldsymbol{n}=366$} & \multicolumn{2}{|c|}{ Savannakhet, $\boldsymbol{n}=369$} & \multicolumn{2}{|c|}{ Luang Prabang, $\boldsymbol{n}=360$} & \multicolumn{2}{|c|}{ Total, $\boldsymbol{n}=1095$} \\
\hline & $\%(n)$ & $\begin{array}{l}\text { Mean EPG } \\
\text { (Max-Min) }\end{array}$ & $\%(n)$ & $\begin{array}{l}\text { Mean EPG } \\
\text { (Max-Min) }\end{array}$ & $\%(n)$ & $\begin{array}{l}\text { Mean EPG } \\
\text { (Max-Min) }\end{array}$ & $\%(n)$ & $\begin{array}{l}\text { Mean EPG } \\
\text { (Max-Min) }\end{array}$ \\
\hline Schistosoma mekongi & $0.5(2)$ & $12(12)$ & $0(0)$ & $0(0)$ & $0(0)$ & $0(0)$ & $0.2(2)$ & $12(12)$ \\
\hline Opisthorchis viverrini & $62.8(230)$ & $\begin{array}{l}2352 \\
(6-41700)\end{array}$ & $77.2(285)$ & $\begin{array}{l}1343 \\
(6-29538)\end{array}$ & $12.2(44)$ & $\begin{array}{l}81 \\
(6-1152)\end{array}$ & $51.1(559)$ & $\begin{array}{l}1659 \\
(6-41700)\end{array}$ \\
\hline Hookworm & $40.4(148)$ & $300(6-5472)$ & $68.6(253)$ & $340(6-6306)$ & $86.4(311)$ & $\begin{array}{l}1207 \\
(6-14004)\end{array}$ & $65.0(712)$ & $\begin{array}{l}710 \\
(6-14004)\end{array}$ \\
\hline Trichuris trichiura & $0.5(2)$ & $18(12-24)$ & $1.4(5)$ & $72(24-108)$ & $31.7(114)$ & $50(6-306)$ & $11.1(121)$ & $50(6-306)$ \\
\hline Ascaris lumbricoides & $0.5(2)$ & $228(78-378)$ & $0.3(1)$ & $12(12)$ & $12.5(45)$ & $\begin{array}{l}5429 \\
(24-71970)\end{array}$ & 4.40 & $\begin{array}{l}5100 \\
(12-71970)\end{array}$ \\
\hline
\end{tabular}

${ }^{a}$ Overall prevalence of Enterobius vermicularis and Taenia spp. was 1.8 and 2.0\%, respectively. EPG: Eggs per gram

\section{POC-CCA test performance}

Overall, 73 participants (6.7\%) had positive POC-CCA tests (Table 3$)$. The highest positivity rate was observed in the $S$. mekongi-endemic Champasack province (11.5\%), while Savannakhet and Luang Prabang had rates of 6.0 and $2.5 \%$, respectively. When trace observations were considered as positive, the positivity rate almost tripled, with a high of $39.1 \%$ in Champasack. Even in Savannakhet and Luang Prabang, where Schistosoma is not endemic, considerably high positivity rates were observed: 14.6 and $7.8 \%$, respectively.

\section{Urine analysis}

The results of the urine analysis to detect urinary tract abnormalities were summarized in Table 4. Overall, onethird $(30.9 \%)$ of the urine samples contained leucocytes. A few participants (3.0\%) tested positive for nitrite. Erythrocytes were detected in about one quarter of the samples (24.5\%), while proteinuria was found in $12.5 \%$ of all urine samples. Eight participants (aged $\geq 12$ years) $(0.7 \%)$ had a positive pregnancy test result.

\section{Association between POC-CCA results and other factors}

The results of the bivariate association between POC-CCA urine test outcomes and various covariates (additional file 1). In the first model, an observed trace counted as a negative result, and in the second model, it counted as a positive result. The strongest associations were observed between the POC-CCA trace-positive outcomes and helminth infections. For example, the association with $O$. viverrini infection was statistically significant, with an $O R$ of 1.65 (95\% CI: $1.22-2.23, P=0.001$ ). The presence of leukocytes and/or nitrite in the urine showed significant positive associations with both trace-negative $(O R=1.78,95 \% C I: 1.10-2.89, P=0018)$ and tracepositive $(O R=1.79, \quad 95 \% \quad C I: \quad 1.32-2.42, \quad P=0.001)$ models. Likewise, the presence of hematuria showed significant positive associations with both tracenegative $(O R=1.67,95 \% C I: 1.01-2.76, P=0.046)$ and trace-positive $(O R=162,95 \% C I: 1.17-2.23, P=0.003)$ models. None of the regression models associated POC-CCA test outcomes with S. mekongi infection. One of the two individuals who tested positive for $S$. mekongi eggs with the KK technique also had a clear

Table 3 POC-CCA test in urine: Performance in diagnosing Schistosoma mekongi in three provinces in Lao PDR $(n=1095)$

\begin{tabular}{|c|c|c|c|c|}
\hline Indicators & $\begin{array}{l}\text { Champasack, } n=366 \\
\%(\boldsymbol{n})\end{array}$ & $\begin{array}{l}\text { Savannakhet, } n=36 \\
\%(n)\end{array}$ & $\begin{array}{l}\text { Luang Prabang, } n=360 \\
\%(n)\end{array}$ & $\begin{array}{l}\text { Total, } n=1059 \\
\%(\boldsymbol{n})\end{array}$ \\
\hline \multicolumn{5}{|c|}{ POC-CCA analysis } \\
\hline Positive & $11.5(42)$ & $6.0(22)$ & $2.5(9)$ & $6.7(73)$ \\
\hline Trace & $27.6(101)$ & $8.7(32)$ & $5.3(19)$ & $13.9(152)$ \\
\hline Negative & $60.9(223)$ & $85.4(315)$ & $92.2(332)$ & $79.5(870)$ \\
\hline \multicolumn{5}{|c|}{ Trace considered as negative } \\
\hline Positive & $11.5(42)$ & $6.0(22)$ & $2.5(9)$ & $6.7(73)$ \\
\hline Negative & $88.5(324)$ & $94.0(347)$ & $97.5(351)$ & $93.3(1022)$ \\
\hline \multicolumn{5}{|c|}{ Trace considered as positive } \\
\hline Positive & $39.1(143)$ & $14.6(54)$ & $7.8(28)$ & $20.5(225)$ \\
\hline Negative & $60.9(223)$ & $85.4(315)$ & $92.2(332)$ & $79.5(870)$ \\
\hline
\end{tabular}


Table 4 Urine analysis to detect urinary tract abnormalities among all study participants

\begin{tabular}{|c|c|c|c|c|}
\hline Parameters & $\begin{array}{l}\text { Champasack, } n=366 \\
\%(\boldsymbol{n})\end{array}$ & $\begin{array}{l}\text { Savannakhet, } n=369 \\
\%(\boldsymbol{n})\end{array}$ & $\begin{array}{l}\text { Luang Prabang, } n=360 \\
\%(\boldsymbol{n})\end{array}$ & $\begin{array}{l}\text { Total, } n=1095 \\
\%(\boldsymbol{n})\end{array}$ \\
\hline \multicolumn{5}{|l|}{ Urinalysis, \% (n) } \\
\hline Leukocyte $(1+, 2+$ and $3+)$ & $33.6(123)$ & $32.8(121)$ & $26.1(94)$ & $30.9(338)$ \\
\hline Nitrite (positive) & $3.6(13)$ & $4.1(15)$ & $1.4(5)$ & $3.0(33)$ \\
\hline Erythrocyte $(1+, 2+, 3+$ and $4+)$ & $23.0(84)$ & $22.8(84)$ & $27.8(100)$ & $24.5(268)$ \\
\hline Protein $(1+, 2+$ and $3+)$ & $5.7(21)$ & $8.7(32)$ & $23.3(84)$ & $12.5(137)$ \\
\hline \multicolumn{5}{|l|}{ Pregnancy test } \\
\hline Positive & $1.1(4)$ & $0.3(1)$ & $0.8(3)$ & $0.7(8)$ \\
\hline
\end{tabular}

positive POC-CCA test, while the other KK-positive individual had a trace-positive POC-CCA result. Of the eight participants who tested positive for pregnancy, one had a clear positive POC-CCA test, while one other had a trace-positive POC-CCA result. Both of these pregnant women originated from Champasack province.

The results of the multivariate analysis of POC-CCA urine test outcomes with all covariates that had a significance level of less than $20 \%$ in the bivariate analysis are shown in Table 5. Again, the analysis was performed for each of the two models, where trace observations were considered as a negative or positive test outcome, respectively. In both models, $O$. viverrini and hookworm infections were significantly associated with the POCCCA test outcomes. Stronger associations were detected in the trace-positive model, particularly between POCCCA positivity and $O$. viverrini infection $(\mathrm{aOR}=1.75$, 95\% CI: 1.29-2.40, $P<0.001$ ) and between POC-CCA negativity and hookworm infection $(\mathrm{aOR}=0.51,95 \% \mathrm{CI}$ : $0.38-0.70, P<0001)$. Hematuria $(\mathrm{aOR}=1.50,95 \% C I$ : 1.07-2.10, $P=0.019)$ and leucocytes/nitrite in urine (aOR $=1.58,95 \% C I: 1.15-2.17, P=0.005)$ were also positively and significantly associated with POC-CCA test outcomes in the trace-positive model; proteinuria was associated with POC-CCA positivity at the $6 \%$ level. No other covariate had a statistically significant association with POC-CCA test outcomes.

Table 5 Multivariate analysis of associations between POC-CCA outcomes and its covariates among study participants in three provinces in Lao PDR $(n=1095)$

\begin{tabular}{|c|c|c|c|c|}
\hline \multirow[t]{2}{*}{ Indicators } & \multicolumn{2}{|c|}{ Trace as negative } & \multicolumn{2}{|l|}{ Trace as positive } \\
\hline & aOR $(95 \%$ CI $)$ & $P$-value & aOR $(95 \%$ Cl $)$ & $P$-value \\
\hline \multicolumn{5}{|l|}{ Helminth infections } \\
\hline O. viverrini & $1.67(1.02-2.77)$ & 0.042 & $1.75(1.29-2.40)$ & $<0.001$ \\
\hline Hookworm & $0.52(0.32-0.85)$ & 0.010 & $0.51(0.38-0.70)$ & $<0.001$ \\
\hline \multicolumn{5}{|l|}{ Urine analysis } \\
\hline Leukocyte/Nitrite & $1.54(0.93-2.54)$ & 0.090 & $1.58(1.15-2.17)$ & 0.005 \\
\hline Hematuria & $1.52(0.90-2.57)$ & 0.119 & $1.50(1.07-2.10)$ & 0.019 \\
\hline Proteinuria & $1.63(0.83-3.17)$ & 0.153 & $1.54(0.99-2.40)$ & 0.056 \\
\hline
\end{tabular}

aOR: Adjusted odds ratio; $\mathrm{Cl}$ : Confidence interval

\section{Discussion}

The POC-CCA test in urine is one of the most efficient tests for diagnosing an active S. mansoni infection, particularly in field settings [7]. It detects circulating cathodic S. mansoni antigens in the urine of infected patients [22]. POC-CCA has been extensively evaluated in areas highly-endemic for S. mansoni. The results of a study performed in five African countries document an overall test sensitivity of $86 \%$ [23], demonstrating that the POC-CCA test has a higher sensitivity compared to the KK technique. Good POC-CCA test sensitivity was also observed in a Swiss study of asymptomatic refugees from a country highly endemic for S. mansoni [9]; the results showed a sensitivity of $91 \%$. Moreover, in areas of Brazil with low S. mansoni endemicity, POC-CCA also showed good sensitivity when trace observations were considered as positive results [24].

Still, evidence from other studies give rise to concerns about POC-CCA test specificity and possible interactions with other conditions, such as urinary tract infection, hematuria [13], and pregnancy [25]. Our previous study of $S$. mekongi-endemic areas in Lao PDR and Cambodia showed an exceedingly high positivity rate with POC-CCA testing, suggesting that other, closely related helminth infections, such as $O$. viverrini, might lead to positive POC-CCA test results [11].

Taking into account these concerns, we aimed to further clarify if the S. mansoni-based POC-CCA test in urine is suitable for diagnosing an active $S$. mekongi infection. In particular, we wanted to clarify the test's specificity in the context of Lao PDR. To do this, we performed a cross-sectional study of children and adults in three provinces in Lao PDR; only one province, Champasack, was known to be endemic for $S$. mekongi.

Overall, we found POC-CCA test positivity rates of 6.7 and $20.5 \%$ if trace observations were considered as negative or positive, respectively. Among the three provinces, S. mekongi-endemic Champasack had the highest positivity rate of $11.5 \%$. However, considerable positivity rates were also observed in Savannakhet and Luang Prabang, 6.0 and 2.5\%, respectively. As neither province is endemic for $S$. mekongi, the results indicate a high rate 
of false-positive POC-CCA test results. When POCCCA trace observations were considered as positive results, the positivity rates tripled.

We found that POC-CCA test results were associated with other helminth infections. In particular, POC-CCA test positivity was positively and significantly associated with $O$. viverrini infection. This observed association is evidence of a likely cross-reaction since $O$. viverrini is a trematode closely related to the Schistosoma species. It is a major public health problem in Lao PDR $[15,16]$, and highly prevalent in the central and southern regions of the country, including Champasack and Savannakhet provinces. Further, POC-CCA test results were also associated with hookworm infection when trace observations were considered as positive results; individuals with positive POC-CCA tests had a lower risk of hookworm infection compared to those with negative POC-CCA tests. This observation is best explained by the geographical concurrence of hookworm infection and not by cross-reactivity. Thus, to confirm the cross-reactivity of POC-CCA test and other helminth infections, an alternative approach can be used for future studies such as pooling of samples with known combinations of parasites or a co-examination with the more specific polymerase chain reaction method.

Previous studies found that POC-CCA tests yield false positive results in patients with urine infections, hematuria, and proteinuria [13]. In our study, we found only partial evidence of these associations. Although these conditions were associated with POC-CCA test results in the univariate analysis, they were only associated in the multivariable analysis when trace observations were considered as positive. This finding might be explained by the fact that, unlike the previous studies, our study was conducted in a community setting and not in a clinic.

Of the eight pregnant women in our study, one tested positive with POC-CCA and another tested trace positive. As these two women originated from Champasack, our study does not provide any evidence that pregnancy leads to false positive POC-CCA results. However, the cross-reaction between POC-CCA and pregnancy has been observed in two other studies. One 2016 study found that two pregnant women from Switzerland, with no history of $S$. mansoni infection, tested positive with POC-CCA [25]. Similarly, a 2019 study from Switzerland [13] showed that 3 out of 15 pregnant women residing in Switzerland tested positive for S. mansoni infection with POC-CCA.

The low number of $S$. mekongi-confirmed patients in our study group makes it difficult to provide a meaningful sensitivity estimate for POC-CCA testing. Over the last decade, annual mass drug administration combined with community awareness programs have reduced $S$. mekongi infection rates to a low level [26]. Our study found only two patients with a confirmed infection, compared to our previous study in 2017 [11], where $6.4 \%(n=24)$ of study participants tested positive with the KK technique. In future studies, we will need to take into account further the low infection rates by examining an increased number of stool samples and smears per stool in order to further increase the sensitivity of KK [27].

\section{Conclusions}

Using POC-CCA tests in areas that are not endemic for S. mekongi leads to many false-positive results in Lao PDR, due to cross-reactivity with $O$. viverrini infection. In the $S$. mekongi-endemic areas of Lao PDR and Cambodia, we recommend caution when using POCCCA testing; positive cases may require more rigorous parasitological testing to confirm the infection.

\section{Supplementary information}

Supplementary information accompanies this paper at https://doi.org/10. 1186/s40249-020-00733-z.

Additional file 1. Bivariate analysis of associations between POC-CCA outcomes and covariates among study participants living in three provinces in Lao PDR $(n=1095)$.

\section{Abbreviations \\ aOR: Adjusted Odds Ratio; Cl: Confidence interval; OR: crude Odds Ratio; EPG: Eggs per gram; KK: Kato-Katz; Lao PDR: Lao People's Democratic Republic; MDA: Mass drug administration; O. viverrini: Opisthorchis viverrini; POC-CCA: Point-of-care circulating cathodic Schistosoma mansoni antigen; PZQ: Praziquantel; STH: Soil-transmitted helminth; S. mekongi: Schistosoma mekongi}

\section{Acknowledgements}

For their various contributions, we thank the staff of the Lao Tropical and Public Health Institute, Vientiane, Lao PDR; the National Center of Mariology, Parasitology and Entomology, Ministry of Health, Vientiane, Lao PDR; and the Provincial Health Departments of Champasack, Savannakhet and Luang Prabang. We also thank the staff at the District Health Offices of Khong, Champhone and Namback for collecting field data and performing KatoKatz, urine dipstick and POC-CCA tests. We are especially grateful to the study participants and authorities at all levels for their support and contributions to our study. Further, we thank Miss Amenda Briet for language corrections.

\section{Authors' contributions}

AH collected and analyzed the data, and drafted the original manuscript. PO formulated the research aims, developed the methodology, provided study materials, supervised the research and critical reviewed and edited the manuscript. PS collected data and coordinated the research. AY critical reviewed and edited the manuscript, and provided financial support for the project. SS formulated the research aims, developed the methodology, provided study materials, supervised the research, analyzed data, and critically reviewed and edited the manuscript. The author(s) read and approved the final manuscript.

\section{Funding}

The World Health Organization, Western Pacific Region, International Development Research Centre, Phase 2 has funded our study, along with the Swiss Agency for Development and Cooperation and the Swiss National Science Foundation (project No. IZ07Z0-160930, R4D). 


\section{Availability of data and materials}

Data is available from the corresponding author upon reasonable request.

\section{Ethics approval and consent to participate}

The study was approved by the National Ethics Committee for Health Research, Ministry of Health (MoH), Vientiane, Lao PDR (Ref.no.113/2018 NECHR). Permission for fieldwork was obtained from the $\mathrm{MoH}$, the Provincial Health Office (PHD), the District Health Office (DHO) and the District Office of Education and Sports. Village and school meetings were held in each study site. Village authorities, villagers, teachers and students were given detailed explanations about the aims, procedures, potential risks, and benefits of the study. A consent and/or assent form in the local language was read to the participants and their questions answered. Signed informed consent was obtained from all study participants before enrollment. For children (under 18 years old), a signed assent was collected and written informed consent was obtained from their parent or quardian. All study participants infected with $O$. viverrini, S. mekongi or soil-transmitted helminths $(\mathrm{STH})$ were treated with praziquantel and albendazole, according to the national guidelines [28].

\section{Consent for publication}

Not applicable.

\section{Competing interests}

The authors declare no competing interests.

\section{Author details}

'Lao Tropical and Public Health Institute, Ministry of Health, Vientiane Capital, Lao People's Democratic Republic. ${ }^{2}$ Department of Epidemiology and Public Health, Swiss Tropical and Public Health Institute, P.O. Box, CH-4002 Basel, Switzerland. ${ }^{3}$ University of Basel, P.O. Box, CH-4003 Basel, Switzerland. ${ }^{4}$ Department of Radiology, Mahosot Hospital, Ministry of Health, Vientiane Capital, Lao People's Democratic Republic. ${ }^{5}$ World Health Organization, Western Pacific Regional Office, Manila, Philippines.

\section{Received: 18 April 2020 Accepted: 29 July 2020}

Published online: 12 August 2020

\section{References}

1. Muth S, Sayasone S, Odermatt-Biays S, Phompida S, Duong S, Odermatt P. Schistosoma mekongi in Cambodia and Lao People's Democratic Republic Zhou XN, Bergquist R, Olveda R, Utzinger J, editers. Elsevier: In; 2010.

2. Dupont V, Bernard E, Soubrane J, Halle B, Richir C. Hepatosplenic form of bilharziasis caused by Schistosoma japonicum manifested by severe hematemesis. Bull Mem Soc Med Hop Paris. 1957;73:933-41.

3. Urbani C, Sinoun M, Socheat D, Pholsena K, Strandgaard H, Odermatt P, et al. Epidemiology and control of mekongi schistosomiasis. Acta Trop. 2002. https://doi.org/10.1016/s0001-706x(02)00047-5.

4. Ohmae H, Sinuon M, Kirinoki M, Matsumoto J, Chigusa Y, Socheat D, et al. Schistosomiasis mekongi: from discovery to control. Parasitol Int. 2004. https://doi.org/10.1016/j.parint.2004.01.004

5. World Health Organization. Meeting Report. In: Expert Consultation to Accelerate Elimination of Asian Schistosomiasis. 2017. https://apps.who.int/ iris/bitstream/handle/10665/259630/RS-2017-GE-36-CHN-eng.pdf?sequence= 1 \&isAllowed $=y$. Accessed 22 June 2019.

6. Barenbold O, Raso G, Coulibaly JT, N'Goran EK, Utzinger J, Vounatsou P. Estimating sensitivity of the Kato-Katz technique for the diagnosis of Schistosoma mansoni and hookworm in relation to infection intensity. PLoS Negl Trop Dis. 2017. https://doi.org/10.1371/journal.pntd.0005953.

7. van Lieshout L, Polderman AM, Deelder AM. Immunodiagnosis of schistosomiasis by determination of the circulating antigens CAA and CCA, in particular in individuals with recent or light infections. Acta Trop. 2000. https://doi.org/10.1016/s0001-706x(00)00115-7.

8. Corstjens PL, van Lieshout L, Zuiderwijk M, Kornelis D, Tanke HJ, Deelder AM, et al. Up-converting phosphor technology-based lateral flow assay for detection of Schistosoma circulating anodic antigen in serum. J Clin Microbiol. 2008. https://doi.org/10.1128/JCM.00877-07.

9. Chernet A, Kling K, Sydow V, Kuenzli E, Hatz C, Utzinger J, et al. Accuracy of diagnostic tests for Schistosoma mansoni infection in asymptomatic Eritrean refugees: serology and point-of-care circulating Cathodic antigen against stool microscopy. Clin Infect Dis. 2017. https://doi.org/10.1093/cid/cix366.
10. World Health Organization. Report of the WHO strategic and technical advisory group. In: The eighth meeting of the strategic and technical advisory Group for Neglected Tropical Diseases (STAG-NTD). 2015. http:// www.who.int/neglected_diseases/NTD_STAG_report_2015.pdf. Accessed 20 Aug 2019 .

11. Vonghachack Y, Sayasone S, Khieu V, Bergquist R, van Dam GJ, Hoekstra PT, et al. Comparison of novel and standard diagnostic tools for the detection of Schistosoma mekongi infection in Lao People's Democratic Republic and Cambodia. Infectious diseases of poverty. 2017;6:1-13.

12. Neumayr A, Chernet A, Sydow V, Kling K, Kuenzli E, Marti H, et al. Performance of the point-of-care circulating cathodic antigen (POC-CCA) urine cassette test for follow-up after treatment of $S$. mansoni infection in Eritrean refugees. Travel Med Infect Dis. 2019; doi:https://doi.org/10.1016/j tmaid.2018.09.004

13. Marti $H$, Halbeisen S, Bausch K, Nickel B, Neumayr A. Specificity of the POCCCA urine test for diagnosing S. mansoni schistosomiasis. Travel Med Infect Dis. 2020; doi:https://doi.org/10.1016/..tmaid.2019.101473.

14. Lao Statistics Bureau. Population and housing census. In: Results of population and housing census 2016. https://lao.unfpa.org/en/publications/ results-population-and-housing-census-2015-english-version. Accessed 11 Mar 2019.

15. Sayasone S, Mak TK, Vanmany M, Rasphone O, Vounatsou P, Utzinger J, et al. Helminth and intestinal protozoa infections, multiparasitism and risk factors in Champasack province, Lao People's Democratic Republic. PLoS Negl Trop Dis. 2011. https://doi.org/10.1371/journal.pntd.0001037.

16. Sayasone S, Vonghajack Y, Vanmany M, Rasphone O, Tesana S, Utzinger J et al. Diversity of human intestinal helminthiasis in Lao PDR. Trans R Soc Trop Med Hyg. 2009. https://doi.org/10.1016/j.trstmh.2008.10.011.

17. Ribas Salvador A, Jollivet C, Morand S, Thongmalayvong B, Somphavong S, Siew CC, et al. Intestinal parasitic infections and environmental water contamination in a rural village of northern Lao PDR. Korean J Parasitol. 2017:55:523-32.

18. Casacuberta-Partal M, Hoekstra PT, Kornelis D, van Lieshout L, van Dam GJ. An innovative and user-friendly scoring system for standardised quantitative interpretation of the urine-based point-of-care strip test (POC-CCA) for the diagnosis of intestinal schistosomiasis: a proof-of-concept study. Acta Trop. 2019. https://doi.org/10.1016/j.actatropica.2019.105150.

19. Ayele B, Erko B, Legesse M, Hailu A, Medhin G. Evaluation of circulating cathodic antigen (CCA) strip for diagnosis of urinary schistosomiasis in Hassoba school children, Afar. Ethiopia Parasite. 2008. https://doi.org/10. 1051/parasite/2008151069

20. Free AH, Rupe CO, Metzler I. Studies with a new colorimetric test for proteinuria. Clin Chem. 1957;3:716-27.

21. Roche. Urinalysis. In: Compendium of urinalysis - urine test strips and microscopy. 2011. https://diagnostics.roche.com/global/en/products/ instruments/combur_chemstripnephurnitur.html. Accessed 15 Dec 2019

22. Rapid Medical Diagnostics. RMD Pamphlet In: RMD Pamphlet for qualitative detection of Bilharzia (Schistosomiasis). 2011. http://www.rapid-diagnostics.com/ downloads/RMD\%20Pamphlet\%202011_06 13\%20.pdf. Accessed 20 Jul 2019.

23. Colley DG, Binder S, Campbell C, King CH, Tchuem Tchuente LA, N'Goran EK, et al. A five-country evaluation of a point-of-care circulating cathodic antigen urine assay for the prevalence of Schistosoma mansoni. Am J Trop Med Hyg. 2013. https://doi.org/10.4269/ajtmh.12-0639.

24. Siqueira LMV, Couto FFB. Taboada D, Oliveira ÁAd, Carneiro NFdF, Oliveira E, et al. performance of POC-CCA ${ }^{\oplus}$ in diagnosis of schistosomiasis mansoni in individuals with low parasite burden. Rev Soc Bras Med Trop. 2016;49:341-7.

25. Greter H, Krauth SJ, Ngandolo BN, Alfaroukh IO, Zinsstag J, Utzinger J. Validation of a point-of-care circulating Cathodic antigen urine cassette test for Schistosoma mansoni diagnosis in the Sahel, and potential cross-reaction in pregnancy. Am J Trop Med Hyg. 2016. https://doi.org/ 10.4269/ajtmh.15-0577.

26. Khieu V, Sayasone S, Muth S, Kirinoki M, Laymanivong S, Ohmae H, et al. Elimination of schistosomiasis mekongi from endemic areas in Cambodia and the lao people's democratic republic: current status and plans. Trop Med Infect Dis. 2019. https://doi.org/10.3390/tropicalmed4010030.

27. Lovis L, Mak TK, Phongluxa K, Aye Soukhathammavong P, Vonghachack Y, Keiser J, et al. Efficacy of praziquantel against Schistosoma mekongi and Opisthorchis viverrini: a randomized, single-blinded dose-comparison trial. PLoS Negl Trop Dis. 2012. https://doi.org/10.1371/journal.pntd.0001726.

28. Ministry of Health Lao PDR. A diagnosis and treatment guideline for the district hospital in Lao PDR. 2nd ed. Vientiane: Ministry of Health; 2004. 